\title{
Microwave imaging with crossed linear arrays
}

\author{
J.M. Gironés \\ L. Jofre \\ M. Ferrando \\ E. de los Reyes \\ Prof. J.Ch. Bolomey
}

Indexing terms: Array processing, Image processing, Microwave systems

\begin{abstract}
The capabilities of a microwave imaging system working in air using two crossed linear arrays are described. The analytic expressions of the focusing procedures are derived, resolution and available volume of reconstruction are discussed. Finally, some experimental images corresponding to two dielectric objects are also shown.
\end{abstract}

\section{Introduction}

An imaging system working at microwave frequencies presents, with regard to optical systems, advantages for the localisation and identification of remote objects or objects located in inaccessible environments. Indeed, a microwave signal can penetrate optically opaque dielectric media [1], a capability which might be useful when the technique presented is applied to noninvasive industrial diagnosis, to the location of concealed or buried objects. It is possible to resolve volumes as small as a $\lambda / 2$ edge cube inside the image object illuminating just one of its sides. So, we have a 3-dimensional vision system with applications in robotics as an artificial vision device. The results obtained show that the system is potentially competitive with optical bidimensional imaging.

Current studies dealing with this field are directed at simplification of the measurement arrangement, optimisation of numerical reconstruction algorithms and definition of new applications.

The imaging system proposed uses a simple arrangement consisting of two crossed linear arrays. We describe a new numerical focusing procedure which allows the recovery of a 3-dimensional object from its scattered field. Different authors have studied similar problems in acoustics and microwaves $[2,3]$. In this work forward scattering from the object is used and the algorithms have been implemented in an experimental system that has produced bidimensional images and cuts of 3dimensional bodies.

\section{Analytical formulation}

The measurement geometry, sketched in Fig. 1, uses $2 N$ elements, $N$ forming a linear transmitter array and the

Paper 5327H (E12, E4, E15, E11), first received 5th August and in revised form 23rd December 1986

Mr. Gironés, Dr. Jofre, Dr. Ferrando and Dr. de los Reyes are with the Department of Electrophysics, Escola Tecnica Superior d'Enginyers de Telecomunicació, Apartat 30002, 08080 Barcelona, Spain

Prof. Bolomey is with the Groupe d'Electromagnetisme, Laboratoire des Signaux et Systémes, Ecole Supérieure d'Electricité, Plateau du Moulon, F-91190 Gif Sur Yvette, France

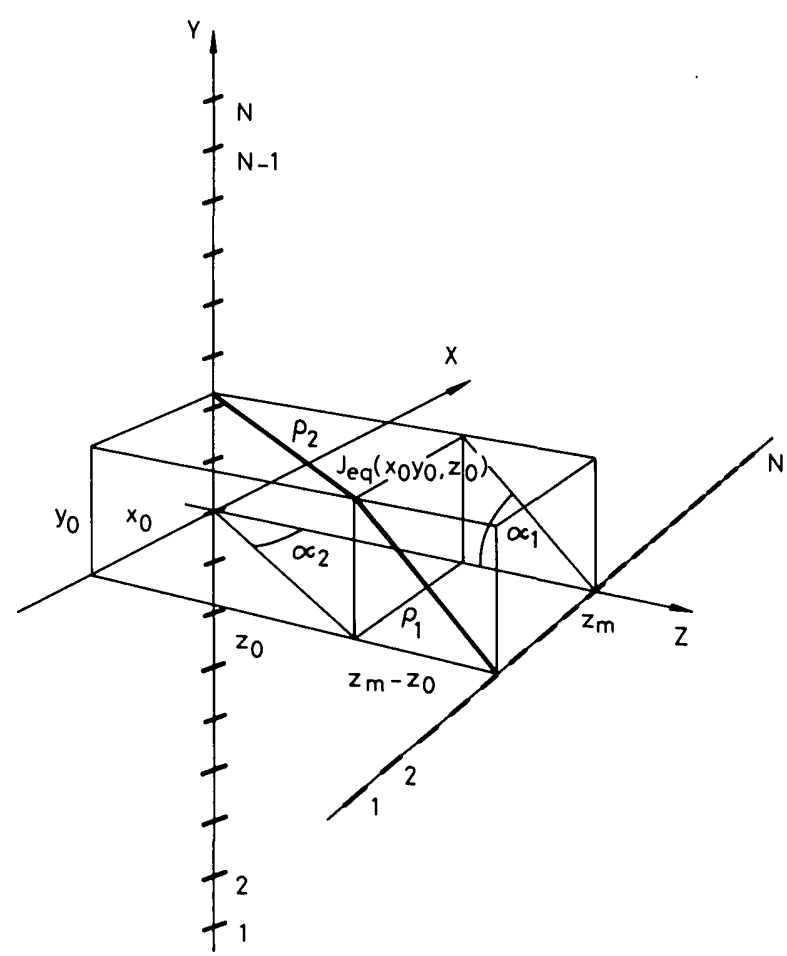

Fig. 1 Measurement geometry for two crossed arrays of 64 elements each, with their centres separated by $16 \lambda$

other $N$ forming an orthogonal linear receiver array separated by a distance $z_{m}$. In this probe arrangement the separation between elements is chosen to be $\lambda / 2$. An $N \times N$ sample matrix, called a conical hologram, is obtained as follows: for every transmitting element selected, the receiver array is scanned obtaining an $N$-sample row, the procedure being repeated for the $N$ elements of the transmitter array. In this way, we are not using physically phased arrays; however, their current distributions are afterwards synthesised by numerical processing of the obtained information.

It is well known that the illumination of an object induces an equivalent electric current distribution, $\boldsymbol{J}_{e q}\left(x_{0}\right.$, $y_{0}, z_{0}$ ), as described in the compensation principle [4]: this distribution forms the electromagnetic image we are interested in recovering. The reconstruction algorithm forms every image points by means of the synthesis of two focused arrays, i.e. all the elements of both arrays are weighted so as to be focused over an unique object point. This is achieved by numerical treatment of the unweighted hologram samples.

The focusing operator can be obtained by inverting the expression of the field created by a current line. Let 
us take the vertical array of Fig. 1; suppose that each element is a magnetic probe (e.g. a dielectric loaded open waveguide) so that we can consider the array as a magnetic current line:

$$
\boldsymbol{M}\left(\boldsymbol{r}^{\prime}\right)=\hat{x} \delta\left(x^{\prime}\right) M_{x}\left(y^{\prime}\right) \delta\left(z^{\prime}\right)
$$

The fields created at a generic point $\boldsymbol{r}$, can be obtained using a spectral formulation of plane and cylindrical waves $[5,6]$, or in a more classical way by means of a vector potential $\boldsymbol{F}$, that satisfies the wave equation

$$
\nabla^{2} \boldsymbol{F}+k^{2} \boldsymbol{F}=-\varepsilon \boldsymbol{M}
$$

where $\varepsilon$ is the permittivity and $k=2 \pi / \lambda$ the wavenumber.

Fourier transforming eqn. 2 with respect to the source variable $y^{\prime}$ results in

$$
\left[\nabla_{t}^{\prime 2}+\left(k^{2}-k_{y}^{2}\right)\right] F T_{y^{\prime}}^{-1}\{\boldsymbol{F}\}=-\varepsilon F T_{y^{\prime}}^{-1}\{\boldsymbol{M}\}
$$

where the transverse Laplacian operator $\nabla_{t}^{\prime 2}$ acts on the $x^{\prime}, z^{\prime}$ components, $k_{y}$ is an angular frequency variable, the operators $F T$ and $F T^{-1}$ denote Fourier transforms, defined by

$$
F T_{y}^{-1}\{\}=\int_{-\infty}^{\infty} e^{-j k_{y} y} d y \quad F T_{k_{y}}\{\}=\frac{1}{2 \pi} \int_{-\infty}^{\infty} e^{j k_{y} y} d k_{y}
$$

The transformed wave equation 3 has the solution

$$
F T_{y^{\prime}}^{-1}\{\boldsymbol{F}\}=\varepsilon \frac{H_{0}^{(2)}\left[\sqrt{ }\left(k^{2}-k_{y}^{2}\right) \rho_{2}\right]}{4 j} F T_{y^{\prime}}^{-1}\{\boldsymbol{M}\}
$$

where $H_{n}^{(2)}$ is the Hankel function of the second kind and $n$ th-order, $\rho_{2}$ the distance from the field point to the current line, as shown in Fig. 1 and $j=\sqrt{ }-1$.

The vertical component of the electric field $\left(E_{y}\right)$, may be obtained directly from $E=-(1 / \varepsilon) \operatorname{rot} F$ and the inverse Fourier transform of eqn. 5:

$$
E_{y}(r)=\frac{j}{4} F T_{k_{y}}\left\{F T_{y^{\prime}}^{-1}\left\{M_{x}\left(y^{\prime}\right)\right\} \frac{\partial}{\partial z} H_{0}^{(2)}\left[\sqrt{ }\left(k^{2}-k_{y}^{2}\right) \rho_{2}\right]\right\}
$$

The $E_{z}$ component is neglected in the reconstruction.

For a given point $\boldsymbol{r}_{0}$, the focusing distribution $M_{x}\left(y^{\prime}\right)$ is the one that makes $E_{y}(r)$ resemble a delta function $\delta\left(\boldsymbol{r}-\boldsymbol{r}_{0}\right)$ as much as possible. In this way we ensure a $y$-selective illumination, obtaining

$$
M_{x}\left(y^{\prime}\right)=4 j F T_{k_{y}}\left\{C\left[k_{y^{\prime}} \rho_{2}\right] e^{j k_{y} y 0}\right\}
$$

where $C\left[k_{y^{\prime}} \rho_{2}\right]$, the focusing operator, is given by

$$
C\left[k_{y^{\prime}} \rho_{2}\right]=\frac{\rho_{2}}{z_{0}} \frac{1}{\sqrt{\left(k^{2}-k_{y}^{2}\right) H_{1}^{(2)}\left[\sqrt{ }\left(k^{2}-k_{y}^{2}\right) \rho_{2}\right]}}
$$

For the horizontal receiver array, proceeding in the same way, we obtain similar expressions. A numerical simultaneous focusing of both arrays over a common point allows to the image point to be obtained:

$$
\begin{aligned}
\xi\left(r_{0}\right)= & \iint_{-\infty}^{\infty} C\left[k_{y^{\prime}} \rho_{2}\right] C\left[k_{x^{\prime}} \rho_{1}\right] F T_{x^{\prime}, y^{\prime}}^{-1}\left\{H_{m}\left(x^{\prime}, y^{\prime}\right)\right\} \\
& \times e^{-j k_{x} x_{0}} e^{-j k_{y} y_{0}} d k_{x} d k_{y}
\end{aligned}
$$

where $H_{m}\left(x^{\prime}, y^{\prime}\right)$ corresponds to the $N \times N$ sample matrix of the magnetic field scattered by the object, and $F T_{x^{\prime}, y^{\prime}}$ is the 2-dimensional generalisation of the Fourier Transforms defined in eqn. 4 .
The previous formulation has been applied to the case of two crossed arrays of $N=64$ antennas each, with a minimum distance between them $z_{m}=16 \lambda$. Simulations have been used to obtain the system impulse response and resolution. We can see, in Fig. 2, the slice $y=0$ of
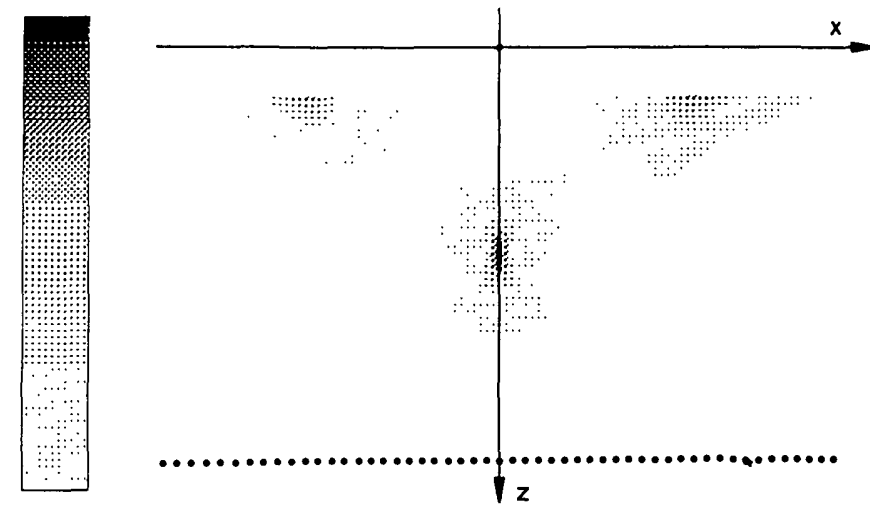

Fig. 2 Image in the $x z$-plane of a point-like object located at $x_{0}=0$, $y_{0}=0, z_{0}=8 \lambda$ (impulse response of the system)

the 3-dimensional reconstruction of a point-like object located at $x_{0}=y_{0}=0, z_{0}=8 \lambda$. The resolution is the same in either the $x$ - or $y$-direction $(\lambda / 2)$ whereas it is $3 \lambda / 2$ in the $z$-direction. Simulations of point-like objects located at points other than the middle of the geometry have shown that the impulse response changes with position, becoming worse as the object moves away from the centre of the geometry. This fact suggests that the available volume of reconstruction should be restricted to a prism of size $10 \lambda \times 20 \lambda \times 20 \lambda$ as shown in Fig. 3 .

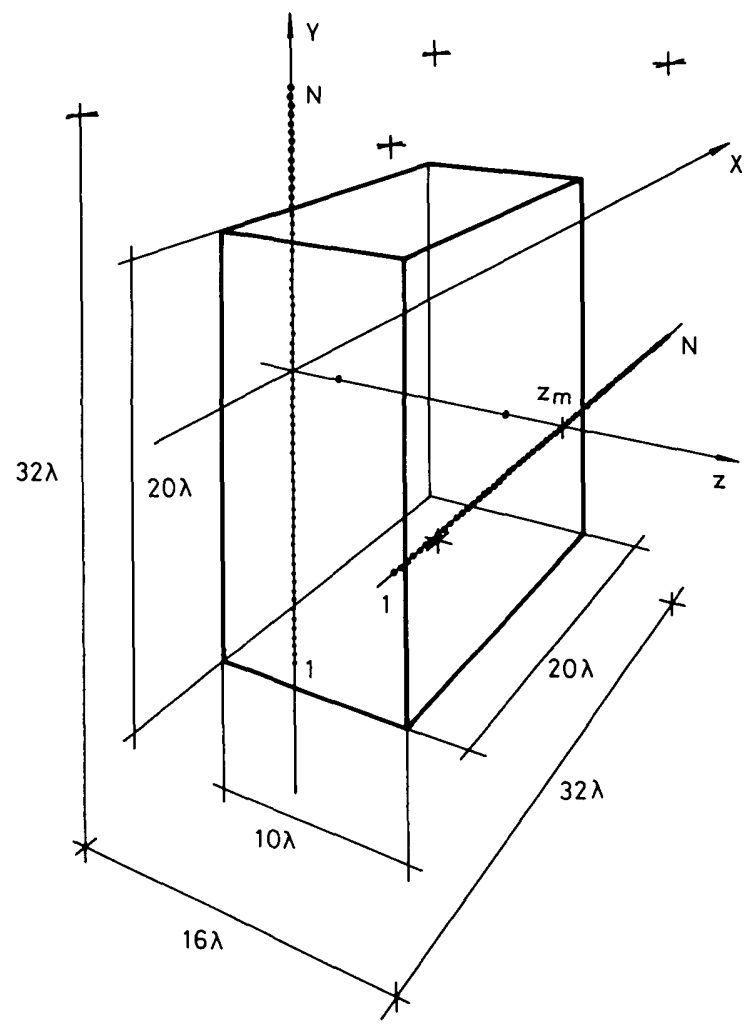

Fig. 3 Available volume of reconstruction $(10 \lambda \times 20 \lambda \times 20 \lambda)$ for the two crossed arrays of lengths $32 \lambda$ separated $16 \lambda$

\section{Experimental results}

The geometry of Fig. 1 has been implemented as illustrated in Fig. 4, the field distribution is obtained by mechanically moving two open waveguides with a system 
controlled by an IEEE-488 bus. A phase-locked sweep oscillator at $12.5 \mathrm{GHz}$, followed by a $1 \mathrm{~W}$ power amplifier, is used as signal generator and an automatic

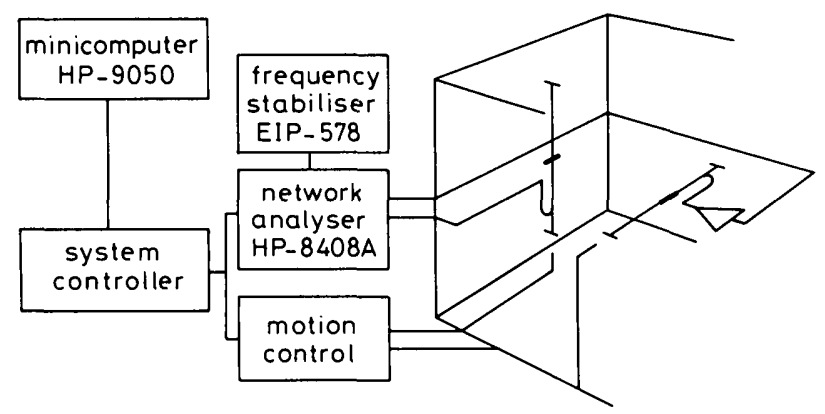

Fig. 4 Experimental set-up with two moving open-ended waveguides that synthesise the arrays
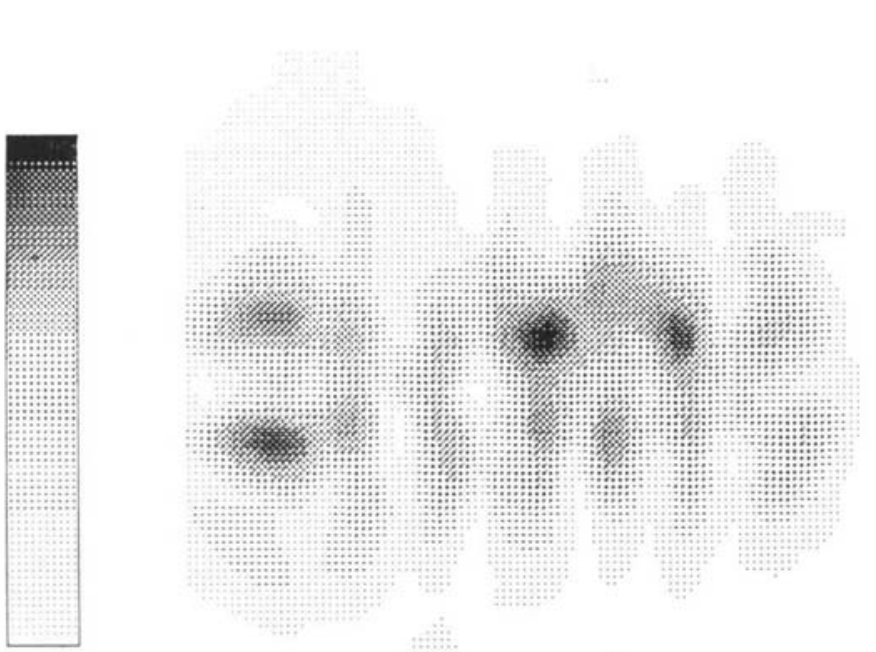

Fig. 5 Field scattered by three dielectric letters $(B M I) 5 \lambda$ high, cut from a $3 \mathrm{~mm}$ thick Teflon sheet

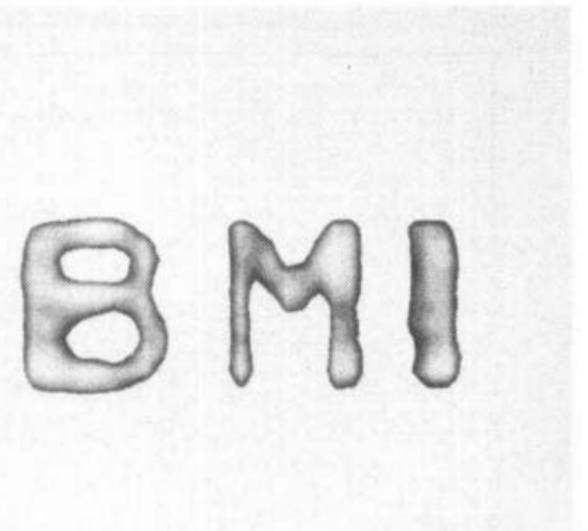

Fig. 6 Reconstruction of the three letter arrangement

network analyser as receiver, permitting a $60 \mathrm{~dB}$ dynamic range with a good phase stability to be obtained.

The scattered field distribution $H_{m}\left(x^{\prime}, y^{\prime}\right)$ is numerically obtained as the difference between the incident (noobject) and the diffracted field. The incident field measurement is used for system calibration and probe compensation. In Fig. 5 we represent the modulus of the field scattered by a 3-letter arrangement. Each of them has been cut out from a Teflon sheet $\left(\varepsilon_{r}=2.1\right)$ and is $5 \lambda$ high. Fig. 6 arises as the result of the application of expr. 9. The reconstruction procedure has not only been applied to almost plane objects but also to 3-dimensional objects. We show, in Figs. 7 and 8, one of the slices that have been obtained by processing an unique matrix $H_{m}\left(x^{\prime}, y^{\prime}\right)$ corresponding to a glass $\left(\varepsilon_{r}=6\right)$ Erlenmeyer flask; it was $7 \lambda$ high and it is sketched in the same Figure.

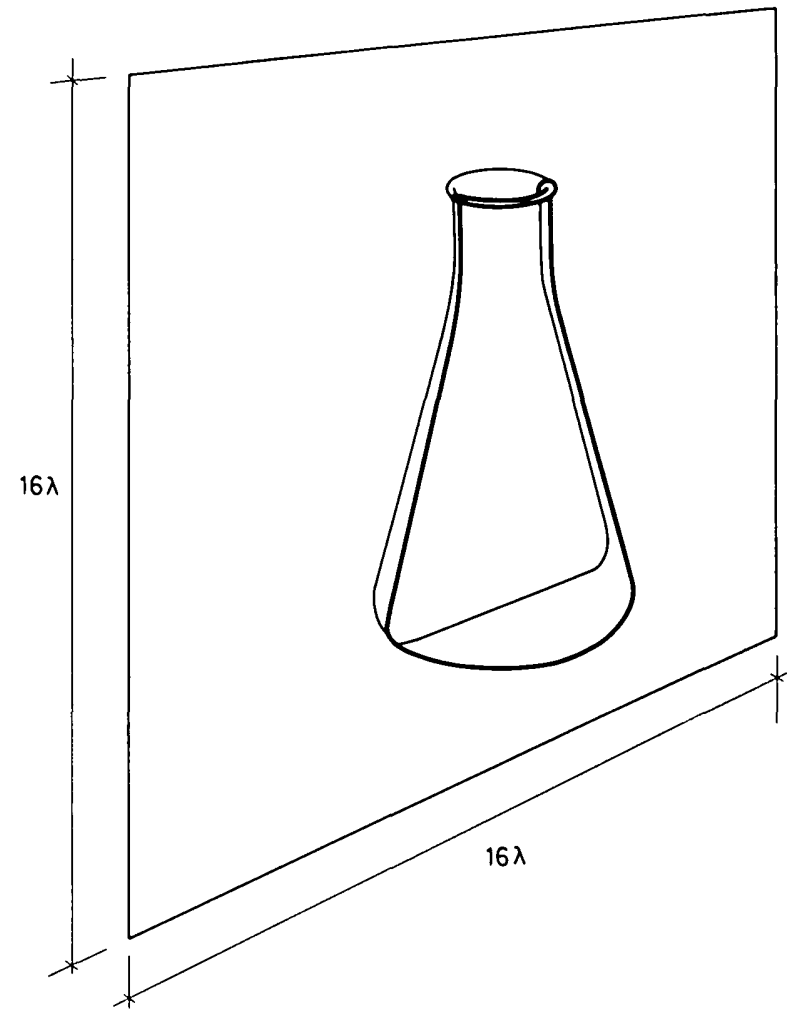

Fig. 7 Erlenmeyer flask

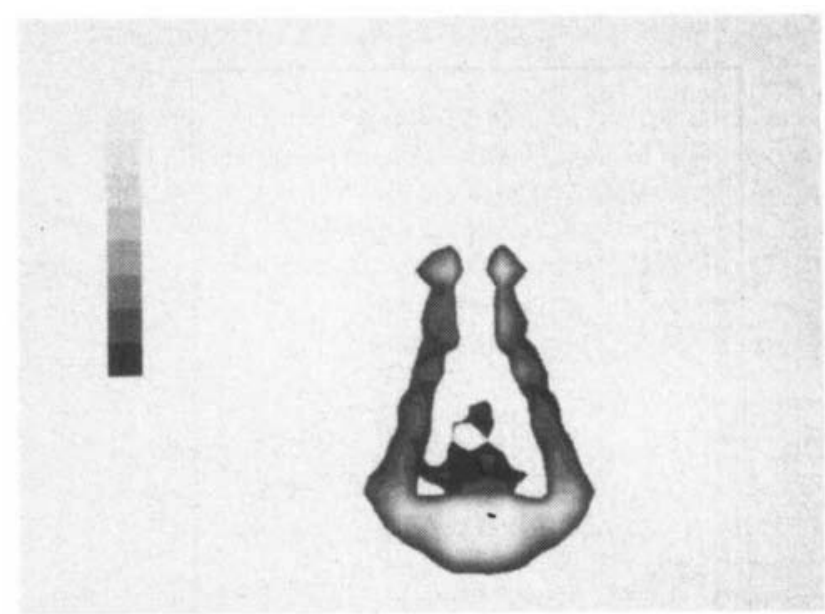

Fig. 8 Image of a central slice

\section{Conclusions}

The quality of the first images obtained with this holographic process shows the possibilities of the proposed method. Obviously, more accurate studies would be necessary to consider a multiview multifrequency realtime set-up, to extract some sort of information from the remaining polarisation or to know the effect of a generic element radiation pattern.

\section{Acknowledgment}

The present work was made possible by the Spanish/ French Committee for Scientific and Technical Research under grant $18 / 133$. 
1 VON HIPPEL, A.: 'Dielectric materials and applications' (MIT Press, Cambridge, MA, 1966)

2 BENNETT, J.C., AL-ADHAMI, S.N.M., and ANDERSON, A.P.: 'A novel radar array and its imaging properties', IEEE Trans., 1979, AP-27, pp. $567-570$

3 PERONNET, G., BOLOMEY, J. Ch., and JOFRE, L.: 'Linear array arrangement for microwave tomography', Radio Sci., 1984, 19, (5), pp. 1342-1346

4 JOFRE, L.: 'Spectral formulation of diffraction problems and its application to microwave imaging'. Doctoral thesis, UPC, Barcelona, 1982 (in Spanish)

5 JORDAN, E.C., and BALMAIN, K.G.: 'Electromagnetic waves and radiating systems' (Prentice hall, Englewood Cliffs, NJ, 1968)

6 MILDER, D.M., and WELLS, W.H.: 'Acoustic holography with crossed linear arrays', IBM J. Res. \& Dev., 1970, 14, pp. 492-500

\section{Errata}

CHRAMIEC, J., and GLASS, A.M.: 'Analysis of microstrip-slotline ring $3 \mathrm{~dB}$ directional couplers', IEE Proc. H, Microwaves, Antennas \& Propag., 1986, 133, (3), pp. $187-190$

The authors would like to point out that the affiliation of A.M. Glass was stated incorrectly. It should have been as follows:

'A.M. Glass was formerly with the Electrical Engineering Department, Basrah University, Basrah, Iraq. He is now with the Department of Electrical \& Electronic Engineering and Applied Physics, Aston University, Aston Triangle, Birmingham B4 7ET, United Kingdom'

$5433 \mathrm{H}$

ALANEN, E., LINDELL, I.V., and HUYANEN, A.T.: 'Exact image method for field calculation in horizontally layered medium above a conducting ground plane', $I E E$ Proc. H, Microwaves, Antenna \& Propag., 1986, 133, (4), pp. 297-304

The authors would like to point out that there is an error of sign concerning the identification of the image currents. In the text at the end of p. 299, $-\partial / \partial x$ should read $\partial / \partial x$. Consequently, the signs in front of the last terms of eqns. 33, 34, 41, and the sign in front of the square brackets in eqn. 66 are erroneous.

$5434 \mathrm{H}$

KORI, M.H., and MAHAPATRA, S.: 'Integral analysis of hybrid coupled semiconductor phase shifters', IEE Proc. H, Microwaves, Antennas \& Propag., 1987, 134, (2), pp. 156-162

In Fig. 3 on p. 158, ' $Z Z_{1}$ ', ' $Z_{2}$ ', ' $Z_{4}$ ' and ' $Z_{3}$ ' should be inserted in the transmission line below ' $\theta_{1}{ }^{\prime},{ }^{\prime} \theta_{2}$ ', ' $\theta_{4}$ ' and ' $\theta_{3}$ ', respectively

On p. 159, in the second paragraph of Section 5.2, ' $Z=50$ ' should read ' $Z=50 \Omega$ '

The 8th line of the same paragraph should read '... the performance $180^{\circ}$ and $90^{\circ}$ phase-shifter bits along with the couplers, are investigated.'

On p. 162, the acknowledgment should read:

'The authors would like to thank the Directorate of Training and Sponsored Research, Defence Research and Development Organisation, The Indian Ministry of Defence, for sponsoring the projection $x$-band pin-diode phase shifters.
RAMANUJAM, P., CLARRICOATS, P.J.B., and BROWN, R.C.: 'Offset spherical reflector with a low sidelobe radiation', IEE Proc. H, Microwaves, Antennas \& Propag., 1987, 134, (2), pp. 199-204

The affiliations of the authors should be as follows:

'Prof. Clarricoats and Mr. Brown are, and Dr. Ramanujam was formerly, with the Department of Electrical \& Electronic Engineering, Queen Mary College, Mile End Road, London E1 4NS, United Kingdom. Dr. Ramanujam is now with the RF Technology Division, ERA Technology, Cleeve Road, Leatherhead, Surrey KT22 7SA, United Kingdom'

In Table 2 on p. 202, the overall height should be $1.35 D$ and not $0.865 D$ as stated

5394H

ANDRES, M.V., and SUCH, V.: 'Cylindrical surface waveguide modes using a surface impedance dyadic method', IEE Proc. H, Microwaves, Antennas \& Propag., 1987, 134, (2), pp. 130-138

On p. 132, in the 3rd line from the bottom of the righthand column, 'from' should be 'for'

On p. 133 , in the line below eqn. $17,{ }^{\prime} F_{1}$ ' should be ' $F_{2}$ ' and vice versa

On the 2nd line of the left-hand column on p. 136, the sentence which begins 'The radial propagation factor ...' should be replaced by '. ..., which are the equations that the radial propagation factor $k$ has to satisfy for large frequencies.'

In eqn. $34 b$, the second ' $W_{n}(y)$ ' should be ' $W_{n}^{\prime}(y)$ '

On the 3rd line of the right-hand column on p. 136, 'fields' should be inserted after 'TE'

In eqns. 42 on p. 138 , the first line of the equation for $E$ should be

$$
E=\int d s\left(\frac{n^{2}}{s} E_{n}^{2}+s E_{n}^{\prime 2}\right)=\left(n+\frac{s^{2}}{2}\right) E_{n}^{2}
$$

Also in eqns. 42 , in the equation

$$
F_{n}(s)=a_{3} J_{n}(s)+a_{4} Y_{n}(s)
$$

' $F_{n}(s)$ ' should be ' $E_{n}(s)$ ' 